\title{
A Passive RFID Sensor Tag for Intelligent Concrete Temperature Control
}

\author{
Zhongbin Chen $^{1, a}$, Fangming Deng ${ }^{2, b}$, Xiang $W^{3, c}$ \\ ${ }^{1}$ School of Electrical and Electronic Engineering, East China Jiao Tong University, Nanchang 330013, \\ China \\ ${ }^{2}$ School of Electrical and Electronic Engineering, East China Jiao Tong University, Nanchang 330013, \\ China \\ ${ }^{3}$ School of Electrical and Electronic Engineering, East China Jiao Tong University, Nanchang 330013, \\ China \\ aemcu00@163.com, bdengfm1980@sina.cn, ${ }^{\mathrm{a}} \mathrm{z}$ gxiangyu@sina.com
}

Keywords: concrete temperature, RF Identification (RFID)

\begin{abstract}
This paper presents an integrated passive sensor tag for intelligent concrete temperature control. The sensor tag is based on EPC Generation-2 UHF communication protocol and operates in passive node. Except the antenna and matching network, the other blocks including RF/analog frontend, digital baseband, electrically erasable programmable read-only memory (EEPROM) and sensors, are fabricated in a single chip for low-cost application, from the test result we can see that this sensor tag can offer a believable measuring result.
\end{abstract}

\section{Introduction}

Concrete material properties change with the time, and these properties (i.e., concrete strength, modulus of elasticity, creep, and shrinkage) are significantly influenced by the heat of hydration and moisture content in the concrete at early ages [1]. Thus, information on the temperature of concrete is significantly important in many areas of civil engineering, especially in the concrete maturity. Curing temperature is one of the parameters considered as a critical factor in the progress of cement hydration by influencing the stability and the transformation of hydrates, and strength development. The result of this process is an increased strength and decreased permeability. High temperature can also promote deterioration processes of concrete structure, therefore, uncontrolled high temperature development during setting and curing of concrete can cause a number of detrimental effects.

Traditional monitoring methods using thermocouples which measure the temperature at the center of the concrete, the high costs and the presence of cables that must be removed when the measurement process is completed which means it is a kind of destructive measurement that limits this technology being widely applied. Thus, the maturation quality of concrete is measured only for some samples [2].

This paper presents a passive wireless temperature sensor tag. Compared with traditional temperature measurement and previous reported sensor designs, the proposed sensor tag has advantages for low-cost and low-power application [2] [3].

\section{Intelligent temperature control method and principle}

To analysis the heat conduction issue of three-dimensional physical properties having internal heat source, according to the Fourier Law in heat transfer theory, the basic heat conduction equation is presented [5]:

$$
\frac{\partial T}{\partial \tau}=a\left(\frac{\partial^{2} T}{\partial x^{2}}+\frac{\partial^{2} T}{\partial y^{2}}+\frac{\partial^{2} T}{\partial z^{2}}\right)+\frac{\dot{Q}}{\rho c}
$$

where T represents temperature, ${ }^{\circ} \mathrm{C}, \tau$ is time variable, d, $\mathrm{c}$ is specific heat capacity, $\mathrm{kJ} /\left(\mathrm{kg} \cdot{ }^{\circ} \mathrm{C}\right)$, $\dot{Q}$ is the heating power of the internal heat source per unit volume, $W / \mathrm{m}^{3}$.

In the overall level of concrete, treating the pipe as internal heat source without considering its space effect in cooling, we can divide the internal heat source into two parts as $\dot{Q}=\dot{Q}^{+}+\dot{Q}^{-}$, where $\dot{Q}^{+}$is the internal heat source caused by the hydration heat of concrete, $\dot{Q}^{-}$is the internal heat 
source caused by pipe endothermic, they can be calculated by the following equations:

$\dot{Q}^{+}=\rho_{c} c_{c} \frac{\partial \theta(\tau)}{\partial \tau}$

$\dot{Q}^{-}=\frac{q_{w} \rho_{w} c_{w} \Delta T(\tau)}{V_{c}}=\frac{q_{w} \rho_{w} c_{w}\left[T_{w-i n}(\tau)-T_{w-\text { out }}(\tau)\right]}{V_{c}}$

where subscript c is concrete, subscript $\mathrm{w}$ is pipe, $T_{w-i n}$ is the input temperature of the pipe and $T_{w-o u t}$ is the output temperature of the pipe, ${ }^{\circ} \mathrm{C}, V_{c}$ is concrete volume, $q_{w}$ is water flow, $\mathrm{L} / \mathrm{min}$, $\theta(t)$ is adiabatic temperature rise function of concrete.

Considering it as steady temperature field, we can get the following equation:

$\frac{\partial T}{\partial \tau}=\frac{\partial \theta(\tau)}{\partial \tau}+\frac{\rho_{w} c_{w}}{\rho_{c} c_{c}} \frac{q_{w}\left[T_{w-i n}(\tau)-T_{w-o u t}(\tau)\right]}{V_{c}}$

Generally, we can get a relatively accurate simulation of the mean temperature field development from equation (4), on the base of temperature prediction we can get the theoretical water flow in equation (5):

$q_{w}(\tau)=\frac{[T(\tau+\Delta \tau)-T(\tau)] / \Delta \tau-\theta^{\prime}(\tau)}{\rho_{w}(\tau) C_{w}(\tau)\left[T_{w-\text { in }}(\tau)-T_{w-\text { out }}(\tau)\right] / \rho_{c} c_{c} V_{c}}$

The overall control process is presented in Fig.1.

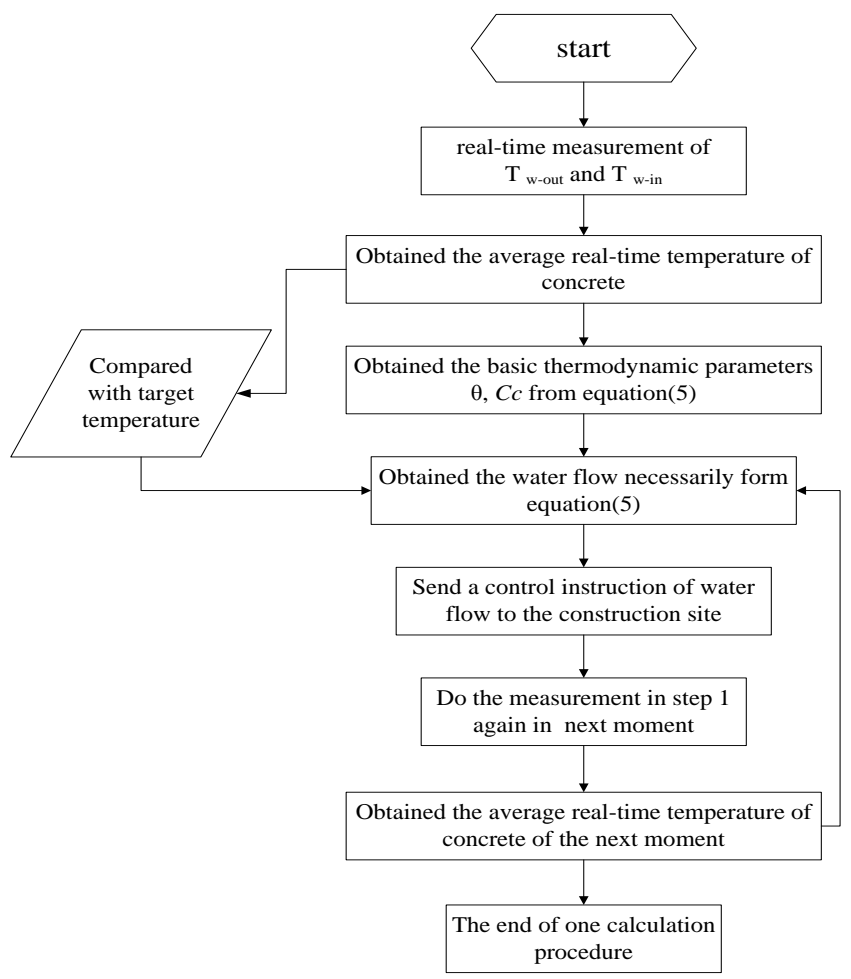

Fig.1 concrete temperature control process

\section{Wireless Sensor Tag Architecture}

Concrete is a mixture of cement, water and aggregated composites. It is well known that the electromagnetic field is highly affected by the dielectric properties of the medium, and the concrete is a lossy material, so its influence on RFID communication should be seriously taken into consideration. Concrete dielectric properties, such as the permittivity $\varepsilon(T ; m ; p ; f ; w / c ; c)$; and the conductivity $\sigma(T ; m ; p ; f ; w / c ; c)$,are generally related to the temperature $T$, the external humidity $m$, the frequency $f$, the time $t$ spent from casting, the location $p$, the water-cement ratio $w / c$ and the type of cement $c$. By fitting data from previous references it is possible to estimate the trend of conductivity and permittivity while the degree of hydration $h_{y}$ changing at UHF frequencies during 
concrete maturation process (Fig. 2), which will be applied in the following numerical analysis. According to Fig. 2, while the conductivity decreases proportionally to the degree of hydration, we can find that the trend of the permittivity is not monotonic and reaches its peak at about one-fifth of the maturation process [5].
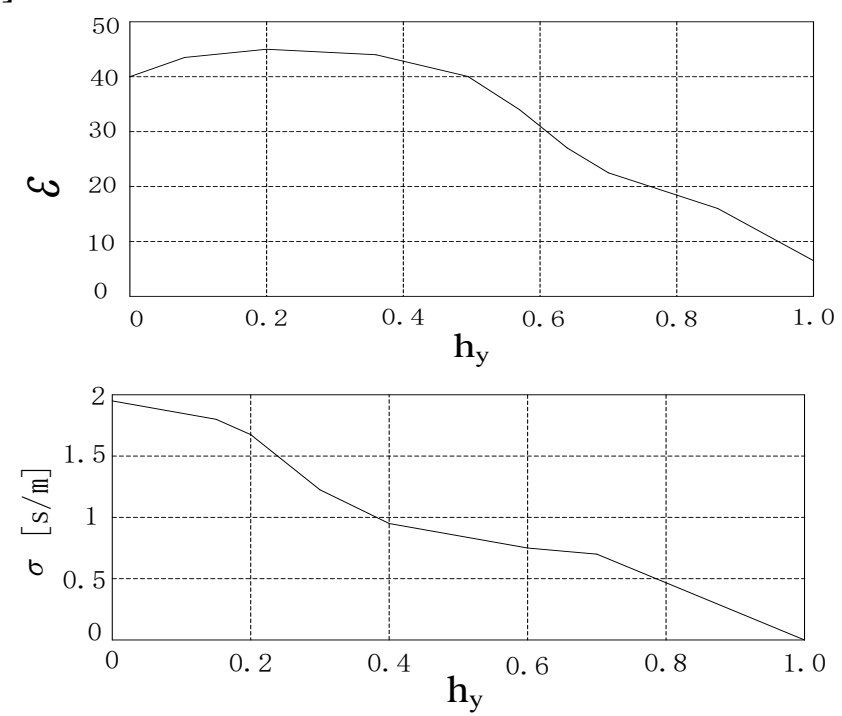

Fig. 2 Top) Permittivity of concrete vs degree of hydration ( $\mathrm{f}=915 \mathrm{MHz}$ );

Bottom) conductivity vs degree of hydration ( $\mathrm{f}=915 \mathrm{MHz})$.

The aim of the proposed RFID sensor is to perform temperature measurements at the center of the concrete, so we should ensure the RFID communication while the sensors inside the concrete [6]. According to the previous reference, we can get a reliable sensor tag design. It is clearly to know that this device completely unreadable from external when the maximum gain of the dipole immersed in concrete is below $-100 \mathrm{~dB}$. So we adapt the proposed T-tag antenna design in Fig. 3 [5] [7].

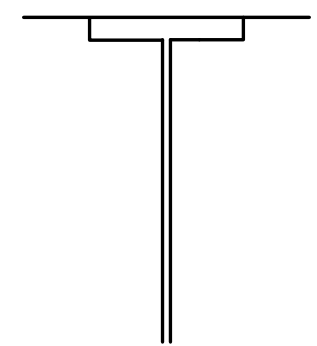

Fig. 3 The proposed antenna

Fig. 4 shows the architecture of the proposed wireless sensor tag. The sensor tag is based on EPC Generation-2 UHF communication protocol and operates in passive node. Except the antenna and matching network, the other blocks including RF/analog frontend, digital baseband, electrically erasable programmable read-only memory (EEPROM) and sensors, are fabricated in a single chip for low-cost application [6] [8].

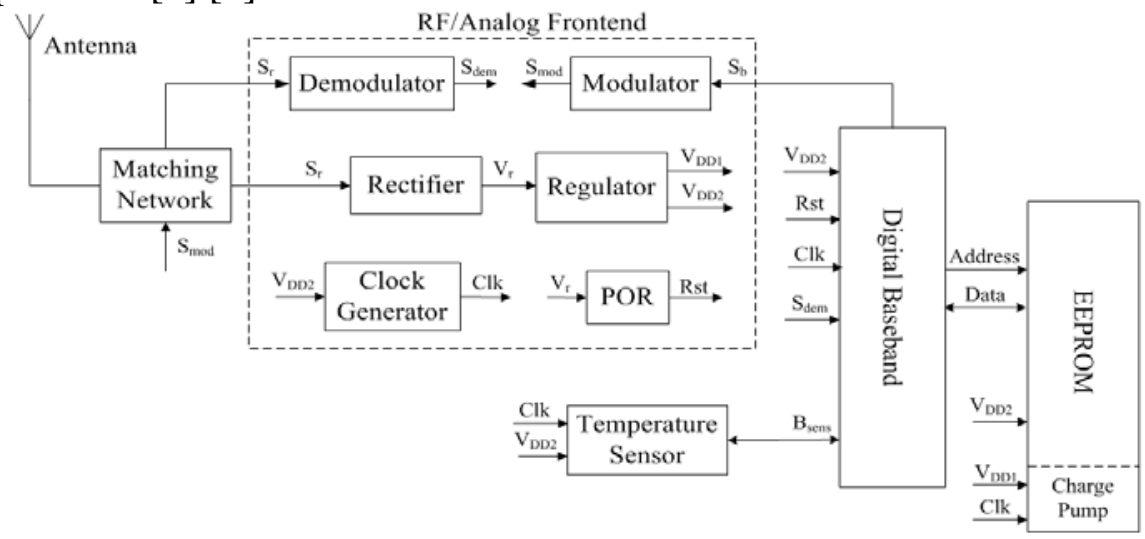

Fig. 4 Architecture of the proposed wireless sensor tag 


\section{Experimental Characterization}

Finally, the temperature measurement capability of the T-tag was tested in reality during the maturation process of fresh concrete. The test points are displayed in Fig. 5, which A is at $15 \mathrm{~cm}$ depth, $\mathrm{B}$ is $10 \mathrm{~cm}$ depth and $\mathrm{C}$ is $5 \mathrm{~cm}$ depth. Three thermocouples were moreover placed in the same position to provide a reference measurement. And one of the T-tags was immersed in a fresh concrete to measure the temperature in realistic conditions (Fig. 7).

After casting, the specimen was exposed to the ambient temperature and the temperature data was recorded every 30 minutes immediately and last for the next 3 days. The specimen is cylindrical with the diameter of $30 \mathrm{~cm}$ and height of $30 \mathrm{~cm}$. Fig. 6 shows the measured temperatures of the RFID sensor and thermocouple as a comparison.

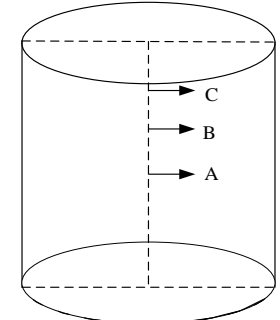

Fig. 5 Test points in specimen

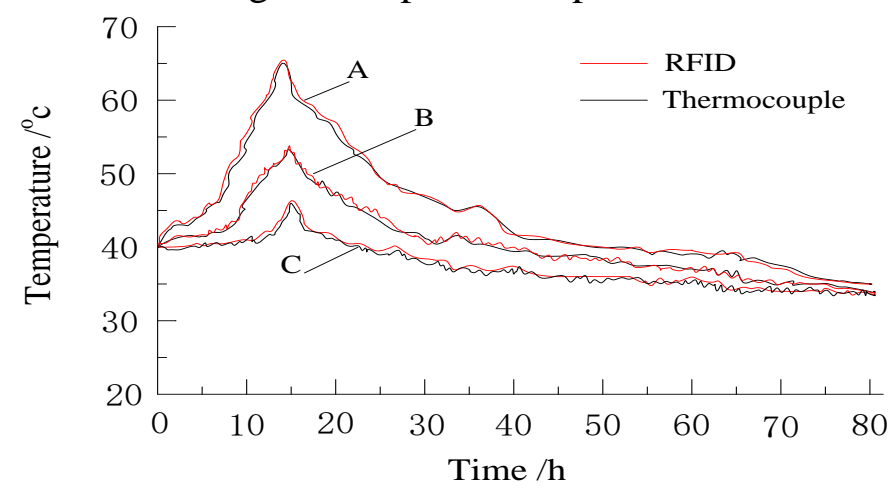

Fig. 6 The measured internal temperature of concrete versus time in two different ways

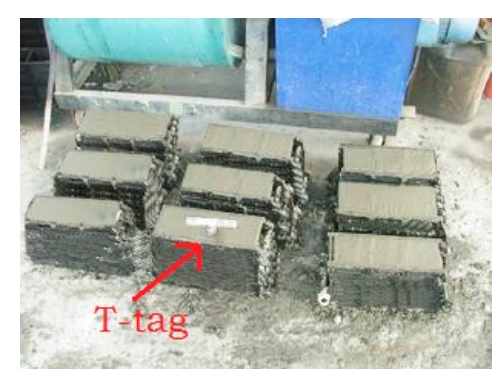

Fig.7 T-tag immersed in a fresh concrete to measure the temperature in realistic conditions

\section{Conclusion}

From Fig. 6 we can see it clearly that the measured temperature by RFID sensor and thermocouple are nearly following the same profile. So the proposed RFID measurement method is believable bringing huge economic benefits, and makes the temperature measurement without destructiveness. The achieved sensor preserves reasonable communication performance suitable for short-range remote sensing all along the drying process and it is able to detect temperature variations inside concrete with a resolution of $0.25^{\circ} \mathrm{C}$. From temperature measurements it is possible to derive fundamental parameters such as the degree of hydration and the mechanical properties. 


\section{Acknowledgements}

This work was supported by East China Jiaotong University Foundation (09DQ06)

\section{References}

[1] Leung C K Y, Pheeraphan T. Microwave curing of Portland cement concrete: experimental results and feasibility for practical applications [J]. Construction and Building Materials, 1995, 9(2): 67-73.

[2] Lynch J P, Loh K J. A summary review of wireless sensors and sensor networks for structural health monitoring [J]. Shock and Vibration Digest, 2006, 38(2): 91-130.

[3] Kim J, Luis R, Smith M S, et al. Concrete temperature monitoring using passive wireless surface acoustic wave sensor system[J]. Sensors and Actuators A: Physical, 2015, 224: 131-139.

[4] $\mathrm{Xu} \mathrm{D}$, Banerjee S, Wang $\mathrm{Y}$, et al. Temperature and loading effects of embedded smart piezoelectric sensor for health monitoring of concrete structures [J]. Construction and Building Materials, 2015, 76: 187-193.

[5] Manzari S, Musa T, Randazzo M, et al. A passive temperature radio-sensor for concrete maturation monitoring[C] RFID Technology and Applications Conference (RFID-TA), 2014 IEEE. IEEE, 2014: 121-126.

[6] Shams K M Z, Ali M. Wireless power transmission to a buried sensor in concrete [J]. Sensors Journal, IEEE, 2007, 7(12): 1573-1577.

[7] Virtanen J, Ukkonen L, Björninen T, et al. Temperature sensor tag for passive UHF RFID systems[C]Sensors Applications Symposium (SAS), 2011 IEEE. IEEE, 2011: 312-317.

[8] Manzari S, Marrocco G. Modeling and applications of a chemical-loaded UHF RFID sensing antenna with tuning capability [J]. Antennas and Propagation, IEEE Transactions on, 2014, 62(1): 94-101. 